\title{
Local Chemical Reactivity of a Metal Alloy Surface
}

\author{
Hammer, Bjørk; Scheffler, Matthias
}

Published in:

Physical Review Letters

Link to article, DOI:

10.1103/PhysRevLett.74.3487

Publication date:

1995

Document Version

Publisher's PDF, also known as Version of record

Link back to DTU Orbit

Citation (APA):

Hammer, B., \& Scheffler, M. (1995). Local Chemical Reactivity of a Metal Alloy Surface. Physical Review Letters, 74(17), 3487-3490. https://doi.org/10.1103/PhysRevLett.74.3487

\section{General rights}

Copyright and moral rights for the publications made accessible in the public portal are retained by the authors and/or other copyright owners and it is a condition of accessing publications that users recognise and abide by the legal requirements associated with these rights.

- Users may download and print one copy of any publication from the public portal for the purpose of private study or research.

- You may not further distribute the material or use it for any profit-making activity or commercial gain

- You may freely distribute the URL identifying the publication in the public portal

If you believe that this document breaches copyright please contact us providing details, and we will remove access to the work immediately and investigate your claim. 


\title{
Local Chemical Reactivity of a Metal Alloy Surface
}

\author{
B. Hammer ${ }^{1,2}$ and M. Scheffler ${ }^{1}$ \\ ${ }^{1}$ Fritz-Haber-Institut der Max-Planck-Gesellschaft, Faradayweg 4-6, D-14195 Berlin-Dahlem, Germany \\ ${ }^{2}$ Center for Atomic-Scale Materials Physics (CAMP) and Physics Department, Technical University of Denmark, \\ DK-2800 Lyngby, Denmark \\ (Received 1 December 1994)
}

\begin{abstract}
The chemical reactivity of a metal alloy surface is studied by density functional theory investigating the interaction of $\mathrm{H}_{2}$ with $\mathrm{NiAl}(110)$. The energy barrier for $\mathrm{H}_{2}$ dissociation is largely different over the $\mathrm{Al}$ and $\mathrm{Ni}$ sites without, however, reflecting the barriers over the single component metal surfaces. This local chemical behavior is due to the covalent nature of the $\left(\mathrm{H}_{2} \sigma_{g}\right)-\left(\mathrm{Ni} 3 d_{z^{2}}\right)$ and $\left(\mathrm{H}_{2} \sigma_{u}^{*}\right)-(\mathrm{Ni}$ $3 d_{x z}$ ) interactions. Thus, it cannot be described in terms of the Harris-Andersson model (i.e., Pauli repulsion and its weakening by empty $d$ states).
\end{abstract}

PACS numbers: 82.65.My, 73.20.At, 73.61.At

Understanding and controlling the physical and chemical mechanisms behind reactions in heterogeneous catalysis stands as one of the long term goals of surface science. Much progress has been made in developing simple, intuitive pictures of how adsorbates interact with solid surfaces $[1,2]$. However, theoretical investigations of the chemical reactivity of alloy and bimetal surfaces is very scarce, despite the fact that many real catalysts consist of solid surfaces of several metallic components [3]. The present Letter starts bridging this gap, by presenting the first density functional theory (DFT) investigation of molecular dissociation on an ordered alloy, namely, $\mathrm{H}_{2}$ on $\mathrm{NiAl}(110)$.

It has been shown previously [4] that the bulk properties of $\mathrm{NiAl}$ are well described in terms of a collective electron band structure with completely filled $\mathrm{Ni} d$ bands. As shown below, the situation is noticeably different at the surface, where the electronic properties at the $\mathrm{Ni}$ and Al sites turn out to be distinguishable. Performing a DFT study of the dissociative adsorption of an $\mathrm{H}_{2}$ molecule on the ordered alloy $\mathrm{NiAl}(110)$ we demonstrate that and explain why the different substrate atoms behave chemically very differently at the surface. The energy barrier against dissociative adsorption is $1.3 \mathrm{eV}$ when the molecule impinges over the $\mathrm{Al}$ site and only $0.5 \mathrm{eV}$ over the $\mathrm{Ni}$ site. Although this difference is significant, the energy barrier over the Ni site remains considerable. This implies that the chemical reactivity is still characteristic of the alloy and not of a clean Ni surface, for which we find no energy barrier. Furthermore, we show that the difference in the energy barriers for $\mathrm{H}_{2}$ dissociation over $\mathrm{Al}$ and $\mathrm{Ni}$ sites can be understood in terms of a covalent interaction of the $\mathrm{H}_{2}$ molecular bonding and antibonding states with the local density of states at the two sites. In particular, we find that the $\left(\mathrm{H}_{2} \sigma_{g}\right)-\left(\mathrm{Ni} 3 d_{z^{2}}\right)$ and $\left(\mathrm{H}_{2} \sigma_{u}^{*}\right)-$ (Ni $3 d_{x z}$ ) interactions ( $z$ being the surface normal and with the $\mathrm{H}_{2}$ axis parallel to the surface and along $x$ ) are strong and have their antibonding combinations partially depopulated. As a consequence, the $\mathrm{H}_{2}$-surface interaction reflects a stronger bonding contribution over the $\mathrm{Ni}$ sites than over the $\mathrm{Al}$ sites. These results are then compared to those for the single component $\mathrm{Ni}(111)$ and $\mathrm{Cu}(111)$ surfaces which also show a significant covalent interaction between the molecule and the surface at close proximity, where the energy barrier occurs. The covalent interaction found in the present Letter has been anticipated by Nørskov and co-workers $[5,6]$ in the construction of $\mathrm{H}_{2}$ metal model potentials. On the other hand, the often celebrated Harris-Andersson concept [7] that empty $d$ states reduce the Pauli repulsion between the molecule and the surface, which certainly applies at larger distances, turns out to be of no relevance in the discussion of whether barriers exist or not as these build up at close distances where the covalent effects dominate.

For the present investigations we choose DFT calculations with the exchange-correlation energy functional of the generalized gradient approximation (GGA) [8] applied non-self-consistently [9] to the charge density found in the local density approximation (LDA) [10]. Similar calculations have proven successful in describing the $\mathrm{H}_{2}$ dissociation on $\mathrm{Cu}(111)$, leading to almost quantitative agreement between calculated and measured barrier heights $[11,12]$.

The ordered alloy $\mathrm{NiAl}$ crystallizes in the $\mathrm{CsCl}$ structure. Its (110) surface thus contains the same number of $\mathrm{Ni}$ and $\mathrm{Al}$ atoms. In our calculations we describe the $\mathrm{NiAl}(110)$ substrate by a slab of five $\mathrm{NiAl}$ layers using the theoretical lattice constant $2.845 \AA$. The geometry of the clean $\mathrm{NiAl}(110)$ surface exhibits a rippled relaxation which is calculated to be $-7.0 \%$ and $+4.5 \%$ of the unrelaxed interlayer separation $(2.0 \AA)$ for the surface $\mathrm{Ni}$ and $\mathrm{Al}$ atoms, respectively $[13,14]$. For the study of the $\mathrm{H}_{2}$-substrate interaction we use a $c(2 \times 2)$ surface cell with two $\mathrm{Ni}$ and two $\mathrm{Al}$ atoms leading to a $\mathrm{H}_{2}-\mathrm{H}_{2}$ separation of $4.9 \AA$, which implies a rather weak interaction between different supercells. The Brillouin zone sampling is performed with 16 special $\mathbf{k}$ points ( $4 \mathbf{k}$ points in the irreducible wedge). The choice of pseudopotentials, plane wave basis, $k T_{e}$, and minimization algorithm is the same as in Ref. [14], where the method proved successful in describing properties of bulk NiAl and its (110) surface. The rippled substrate surface is kept frozen during the interaction with the $\mathrm{H}_{2}$ 
molecule, because a geometry change would happen on a slower time scale than the dissociation event. We note, however, that as the relaxations of the clean and $\mathrm{H}$ covered $\mathrm{NiAl}(110)$ are noticeably different [14], the $\mathrm{H}_{2} / \mathrm{NiAl}(110)$ system might show similar phenomena in associative desorption as found for $\mathrm{H}_{2}$ at $\mathrm{Si}(100)[15,16]$.

Figure 1(a) presents the potential energy curves of the dissociation of an $\mathrm{H}_{2}$ molecule held parallel to the surface and being centered over the $\mathrm{Al}$ and $\mathrm{Ni}$ sites, respectively [Figs. 1(b) and 1(c)]. The in-plane orientation of the $\mathrm{H}_{2}$ molecule is chosen so that both pathways lead directly into the equilibrium $(1 \times 1)$ structure of dissociated atomic hydrogen in the $\mathrm{Ni}-\mathrm{Ni}$ bridge [Fig. 1(d)] reported in Ref. [14]. By considering two pathways with the same as-dissociated hydrogen positions, we obtain potential energy curves that reflect the differences in molecule-surface interaction most clearly. Inspection of the intramolecular forces suggests that over the Al sites, the transition state is at $1.57<Z<1.75 \AA$, while over the $\mathrm{Ni}$ sites it is at $1.19<Z<1.39 \AA$. The energy barrier for $\mathrm{H}_{2}$ dissociation over the $\mathrm{Al}$ site is therefore about $1.3 \mathrm{eV}$ over the $\mathrm{Al}$ site while about $0.5 \mathrm{eV}$ over the $\mathrm{Ni}$ site. This demonstrates that the molecule-surface interaction depends most sensitively on the point of impact. Judging from the sizable activation barrier over the $\mathrm{Ni}$ sites we note that the interaction does not reflect the behavior of a pure Ni surface, for which a vanishing barrier would be expected from experimental observations [17] and for which no barrier is found in the DFT-GGA calculation for $\mathrm{Ni}(111)$ reported below.

In order to elucidate the mechanism behind the different interactions at the two sites, we consider the density of

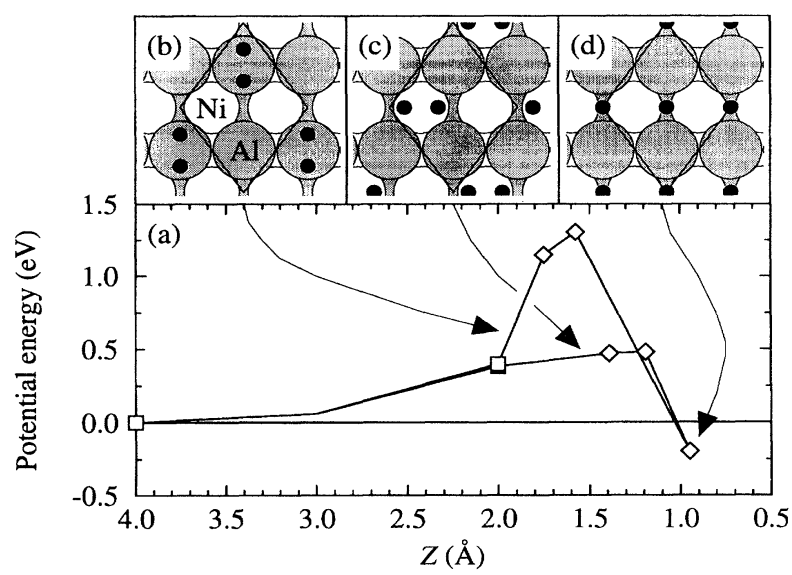

FIG. 1. (a) The potential energy of $\mathrm{H}_{2}$ dissociating over $\mathrm{NiAl}(110)$ versus the height $Z$ of the $\mathrm{H}_{2}$ center of mass from the plane of the unrelaxed surface atomic position. The upper (lower) curve is a guide to the eye connecting points for $\mathrm{H}_{2}$ over the $\mathrm{Al}(\mathrm{Ni})$ site. The geometries have been optimized with respect to the H-H separation $b$ at fixed $Z$ ( $\square$ ) or with respect to $Z$ at fixed $b(\diamond)$. (b) $\mathrm{H}_{2}$ (• pairs) over the $\mathrm{Al}$ sites (shaded circles) of the $\mathrm{NiAl}(110)$. The $c(2 \times 2)$ unit cell is indicated. (c) $\mathrm{H}_{2}$ over the $\mathrm{Ni}$ sites (open circles). (d) $(1 \times 1)$ $\mathrm{H} / \mathrm{NiAl}(110)$. states (DOS) at the energy $\varepsilon$ (relative to the Fermi level) projected on the localized orbital $\phi_{a}$ :

$$
n_{a}(\varepsilon)=\sum_{i} \int\left|\left\langle\phi_{a} \mid \psi_{i}(\mathbf{k})\right\rangle\right|^{2} \delta\left[\varepsilon-\varepsilon_{i}(\mathbf{k})\right] d \mathbf{k},
$$

where $i$ is the band index running over all bands, $\mathbf{k}$ is integrated over the entire Brillouin zone $(\mathrm{BZ}), \psi_{i}(\mathbf{k})$ are the Kohn-Sham wave functions, and $\varepsilon_{i}(\mathbf{k})$ are the corresponding single particle energies. Because of the finite k-point sampling in our calculation, the BZ integral is replaced with a discrete sum, and the $\delta$ function is for convenience replaced with a Gaussian of width $0.25 \mathrm{eV}$.

For $\phi_{a}$ we choose the $\mathrm{H}_{2}$ molecular bonding $\left(\sigma_{g}\right)$ and antibonding $\left(\sigma_{u}^{*}\right)$ orbitals that are constructed as normalized linear combinations of the hydrogen $s$ orbitals [18], $\phi_{s}^{\mathrm{H}}$, centered at the positions of the two hydrogen atoms $\mathbf{R}_{1}$ and $\mathbf{R}_{2}$ :

$$
\begin{aligned}
& \phi_{\sigma_{g}}(\mathbf{r})=c_{1}\left\{\phi_{s}^{\mathrm{H}}\left(\mathbf{r}-\mathbf{R}_{1}\right)+\phi_{s}^{\mathrm{H}}\left(\mathbf{r}-\mathbf{R}_{2}\right)\right\}, \\
& \phi_{\sigma_{u}^{*}}(\mathbf{r})=c_{2}\left\{\phi_{s}^{\mathrm{H}}\left(\mathbf{r}-\mathbf{R}_{1}\right)-\phi_{s}^{\mathrm{H}}\left(\mathbf{r}-\mathbf{R}_{2}\right)\right\},
\end{aligned}
$$

where $c_{1}$ and $c_{2}$ are chosen to ensure normalization.

In Figs. 2 and 3 the projected DOS, $n_{\sigma_{g}}(\varepsilon)$ and $n_{\sigma_{u}^{*}}(\varepsilon)$, are shown for five different positions along the two pathways of Fig. 1. For both pathways, the $\mathrm{H}_{2}$ bonding level is seen to initially broaden and shift down in energy when the molecule approaches the surface. This behavior is readily understood within a Newns-Anderson model [19] as originating from weak coupling of an adsorbate level in resonance with a broad continuum of states, and has been demonstrated in jellium calculations by Lundqvist and coworkers [5,20]. When the molecular bond is succeedingly weakened, the $\mathrm{H}_{2}$ bonding level shifts up and finally meets the antibonding level. In addition to this main feature, also a smaller resonance is observed. This appears in Figs. 2(b) and 3(b) just below the Fermi energy and moves up through the Fermi level as the dissociation is completed. This resonance has antibonding character between the molecule and the surface, as will be apparent below.

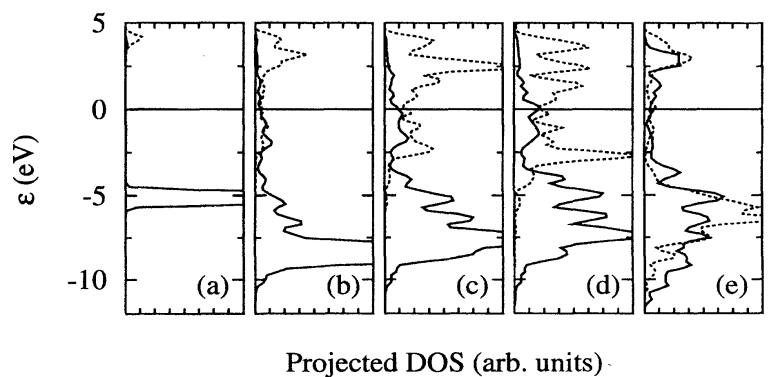

FIG. 2. $\mathrm{H}_{2}$ dissociation over the $\mathrm{Al}$ : Molecular bonding $n_{\sigma_{g}}(\varepsilon)$ (solid curve) and antibonding, $n_{\sigma_{u}^{*}}(\varepsilon)$, (dashed curve) projected DOS at the five positions indicated in Fig. 1(a). Dissociation proceeds from left to right. The $(b, Z)$ coordinates are (a) $(0.78,4.0 \AA)$, (b) $(0.81,2.0 \AA)$, (c) $(1.2,1.75 \AA)$, (d) $(1.5,1.57 \AA)$, and (e) $(2.845,0.95 \AA)$. 


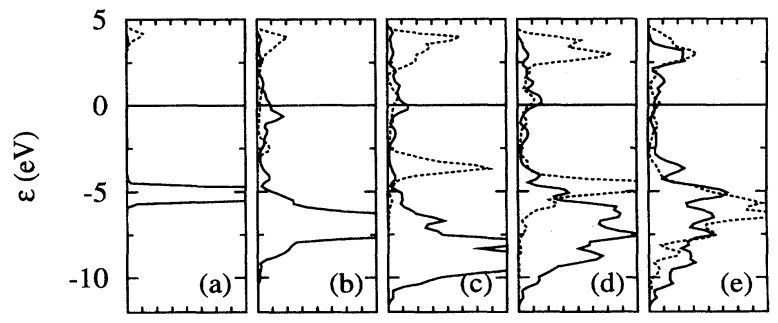

Projected DOS (arb. units)

FIG. 3. Same as Fig. 2 but for $\mathrm{H}_{2}$ dissociation over the Ni. The $(b, Z)$ coordinates are (a) $(0.78,4.0 \AA)$, (b) $(0.81,2.0 \AA)$, (c) $(1.0,1.39 \AA)$, (d) $(1.5,1.19 \AA)$, and (e) $(2.845,0.95 \AA)$.

The interaction of the $\mathrm{H}_{2} \quad \sigma_{u}^{*}$ orbital with the metal states shows up in Figs. 2 and 3 noticeably different over the two different sites. For the $\mathrm{H}_{2}$ over the Al site, mainly a broad $n_{\sigma_{u}^{*}}$ resonance (dashed curve in Fig. 2) evolves. This can be understood as due to the interaction with a broad continuum of substrate states. For $\mathrm{H}_{2}$ over the Ni site, however, two well-separated narrow peaks evolve in $n_{\sigma_{u}^{*}}$ (dashed curve in Fig. 3). This is the signature of strong covalent coupling between an adsorbate level and a narrow continuum.

In order to achieve information about the chemical bonding character of the individual resonances in Figs. 2 and 3 we proceed by looking at the DOS projected onto three-center orbitals. We define

$$
\begin{gathered}
\xi\left(\mathrm{H}_{2} \sigma_{u}^{*}, \mathrm{Ni} 3 d_{x z}\right)(\mathbf{r})=c_{3}\left[\phi_{\sigma_{u}^{*}}(\mathbf{r})+\chi \phi_{3 d_{x z}}^{\mathrm{Ni}}\left(\mathbf{r}-\mathbf{R}_{\mathrm{Ni}}\right)\right], \\
\xi^{*}\left(\mathrm{H}_{2} \sigma_{u}^{*}, \mathrm{Ni} 3 d_{x z}\right)(\mathbf{r})=c_{4}\left[\phi_{\sigma_{u}^{*}}(\mathbf{r})-\chi \phi_{3 d_{x z}}^{\mathrm{Ni}}\left(\mathbf{r}-\mathbf{R}_{\mathrm{Ni}}\right)\right],
\end{gathered}
$$

where $\phi_{3 d_{x z}}^{\mathrm{Ni}}$ is the atomic $\mathrm{Ni} 3 d_{x z}$ orbital [18], $\mathbf{R}_{\mathrm{Ni}}$ is the position of the surface $\mathrm{Ni}$ atom, $\chi=\operatorname{sgn}\left(\left\langle\phi_{\sigma_{u}^{*}} \mid \phi_{3 d_{x z}}^{\mathrm{Ni}}\right\rangle\right)$, and $c_{3}$ and $c_{4}$ are normalization constants. Orbitals being bonding $(\xi)$ and antibonding $\left(\xi^{*}\right)$ between the molecule and the surface, but involving other $\mathrm{Ni}$ or $\mathrm{Al}$ atomic orbitals, are defined analogously to Eq. (3).

Figures 4 and 5 display the DOS of Figs. 2(c) and 3(c), respectively, projected onto three-center orbitals as defined above. The chemical nature of the individual DOS peaks in Figs. 2 and 3 can now be addressed. The small resonance near the Fermi level in $n_{\sigma_{g}}(\varepsilon)$ is of $\xi^{*}\left(\mathrm{H}_{2} \sigma_{g}, \mathrm{Al} 3 \mathrm{~s}\right)$ character over the Al site and of $\xi^{*}\left(\mathrm{H}_{2} \sigma_{g}, \mathrm{Ni} 3 d_{z^{2}}\right)$ character over the $\mathrm{Ni}$ site. It is seen that this molecule-surface antibonding resonance has a large share of its weight above the Fermi level when the dissociation takes place over the $\mathrm{Ni}$ site. This means that the molecule interacts in a stronger bonding manner with the surface when approaching over the $\mathrm{Ni}$ site than over the Al site. Consequently, the approach over the $\mathrm{Ni}$ site is energetically preferred.

The detailed behavior of $n_{\sigma_{u}^{*}}$ is also revealed by Figs. 4 and 5. Over the $\mathrm{Al}$ site the small peaks at -2 and $4 \mathrm{eV}$ overlaying the main resonance at $2.5 \mathrm{eV}$ are seen to orig-

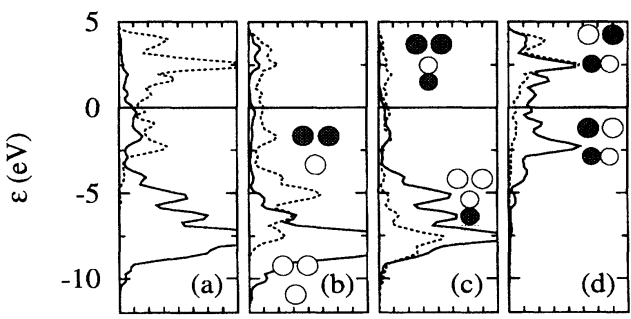

Projected DOS (arb. units)

FIG. 4. $\mathrm{H}_{2}$ over the $\mathrm{Al}$ at $(b, Z)=(1.2,4.0 \AA)$ : The solid and dashed curves, respectively, show the DOS projected onto (a) $\phi_{\sigma_{g}}$ and $\phi_{\sigma_{u}^{*}}$, (b) $\xi\left(\mathrm{H}_{2} \sigma_{g}, \mathrm{Al} 3 s\right)$ and $\xi^{*}\left(\mathrm{H}_{2} \sigma_{g}, \mathrm{Al} 3 s\right)$, (c) $\xi\left(\mathrm{H}_{2} \sigma_{g}\right.$, Al $\left.3 p_{z}\right)$ and $\xi^{*}\left(\mathrm{H}_{2} \sigma_{g}\right.$, Al $\left.3 p_{z}\right)$, and (d) $\xi\left(\mathrm{H}_{2} \sigma_{u}^{*}, \mathrm{Al} 3 p_{y}\right)$ and $\xi^{*}\left(\mathrm{H}_{2} \sigma_{u}^{*}\right.$, Al $\left.3 p_{y}\right)$. In the symbolic sketches of the orbitals the two hydrogen $s$ orbitals are depicted atop a substrate state. White (gray) represents positive (negative) sign.

inate from the splitting of the $\xi\left(\mathrm{H}_{2} \sigma_{u}^{*}\right.$, Al $\left.3 p_{y}\right)$ and the $\xi^{*}\left(\mathrm{H}_{2} \sigma_{u}^{*}, \mathrm{Al} 3 p_{y}\right)$ DOS. Over the Ni site it is the splitting of the $\xi\left(\mathrm{H}_{2} \sigma_{u}^{*}, \mathrm{Ni} 3 d_{x z}\right)$ and the $\xi^{*}\left(\mathrm{H}_{2} \sigma_{u}^{*}\right.$, Ni $\left.3 d_{x z}\right)$ DOS which completely dominates $n_{\sigma_{u}^{*}}$. Again, the degree to which the molecule-surface interaction (now through the intramolecular antibonding level) is bonding determines which reaction pathway is energetically preferred for dissociation. The stronger splitting over the Ni site therefore facilitates the dissociation. The present argumentation is independent of whether the $\mathrm{Ni} 3 d$ states involved in the molecule-surface interaction are initially filled or empty. Thus, while asymptotically for large distances the interaction between a closed shell molecule and a metal surface may be affected by the Pauli repulsion between the filled molecular and substrate orbitals and the presence of a high and empty DOS at the Fermi level (as described by Harris and Andersson [7]) this effect is not important at distances close to the surface, where the dissociation energy barriers are typically found.

In order to complement the findings of the importance of the $\left(\mathrm{H}_{2} \sigma_{g}\right)-\left(\mathrm{Ni} 3 d_{z^{2}}\right)$ and $\left(\mathrm{H}_{2} \sigma_{u}^{*}\right)-\left(\mathrm{Ni} 3 d_{x z}\right)$ interactions on $\mathrm{NiAl}(110)$, we have performed DFT-GGA calculations for $\mathrm{H}_{2}$ dissociation over $\mathrm{Ni}(111)$ and $\mathrm{Cu}(111)$.

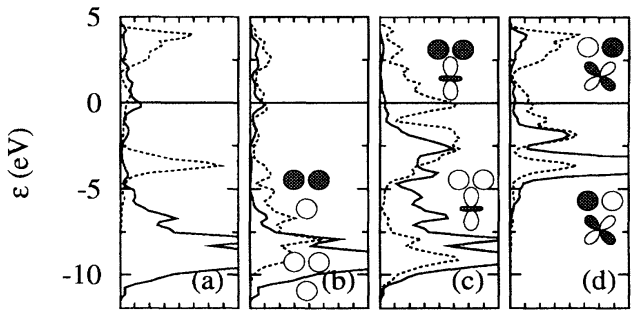

Projected DOS (arb. units)

FIG. 5. $\quad \mathrm{H}_{2}$ over the $\mathrm{Ni}$ at $(b, Z)=(1.0,1.39 \AA)$ : The solid and dashed curves, respectively, show the DOS projected onto (a) $\phi_{\sigma_{g}}$ and $\phi_{\sigma_{u}^{*}}$, (b) $\xi\left(\mathrm{H}_{2} \sigma_{g}, \mathrm{Ni} 4 s\right)$ and $\xi^{*}\left(\mathrm{H}_{2} \sigma_{g}, \mathrm{Ni} 4 s\right)$, (c) $\xi\left(\mathrm{H}_{2} \sigma_{g}, \mathrm{Ni} 3 d_{z^{2}}\right)$ and $\xi^{*}\left(\mathrm{H}_{2} \sigma_{g}, \mathrm{Ni} 3 d_{z^{2}}\right)$, and (d) $\xi\left(\mathrm{H}_{2} \sigma_{u}^{*}\right.$, Ni $\left.3 d_{x z}\right)$ and $\xi^{*}\left(\mathrm{H}_{2} \sigma_{u}^{*}\right.$, Ni $\left.3 d_{x z}\right)$. 
For the geometry we choose $\mathrm{H}_{2}$ parallel to the surfaces, centered over the $\mathrm{Ni}$ and $\mathrm{Cu}$ atoms, respectively, and adsorbing dissociatively directly into the threefold hollow sites of a $(\sqrt{3} \times \sqrt{3})$ surface cell on a 4-layer slab (54 $\mathbf{k}$ points in the BZ). While the energy barrier against dissociation is $0.5 \mathrm{eV}$ on $\mathrm{Cu}(111)$ [11] it is absent on $\mathrm{Ni}(111)$.

Figures 6 and 7 show the $\mathrm{H}_{2} \sigma_{g}$ and $\mathrm{H}_{2} \sigma_{u}^{*}$ projected DOS over $\mathrm{Ni}(111)$ and $\mathrm{Cu}(111)$. Clearly, over both surfaces the $\sigma_{u}^{*}$ projected DOS evolves into two well-separated narrow peaks. Also apparent is that a small peak in $n_{\sigma_{g}}(\varepsilon)$ evolves and goes through the Fermi level as the dissociation proceeds. The major difference between the dissociation on the $\mathrm{Ni}(111)$ and the $\mathrm{Cu}(111)$ can be summarized as follows. On $\mathrm{Ni}$, the $\xi^{*}\left(\mathrm{H}_{2} \sigma_{u}^{*}, \mathrm{Ni} 3 d_{x z}\right)$ DOS is almost empty all along the reaction and the $\xi^{*}\left(\mathrm{H}_{2} \sigma_{g}, \mathrm{Ni} 3 d_{z^{2}}\right)$ DOS goes through the Fermi level at an early stage. On $\mathrm{Cu}$, on the other hand, the $\xi^{*}\left(\mathrm{H}_{2} \sigma_{u}^{*}, \mathrm{Cu} 3 d_{x z}\right)$ DOS has a considerable filling all along the reaction and the $\xi^{*}\left(\mathrm{H}_{2} \sigma_{g}, \mathrm{Cu} 3 d_{z^{2}}\right)$ DOS remains largely filled. This difference arises due to the difference in the positions of the substrate $d$ levels relative to the Fermi level on the two surfaces [Figs. 6(a) and 7(a)] and has as a consequence that $\mathrm{Ni}$ acts with much more molecule-surface bonding character than does $\mathrm{Cu}$. Thus covalent effects involving the molecular states and the metal $d$ states explain the calculated and observed differences in barrier heights. That the same behavior appears to be consistent with the Harris-Andersson picture is only because the energetically higher $\mathrm{Ni} 3 d$ bands compared to the $\mathrm{Cu} 3 d$ bands lead to $d$ holes for $\mathrm{Ni}$ compared to none for $\mathrm{Cu}$. However, as shown for $\mathrm{H}_{2}$ over $\mathrm{NiAl}(110)$ even completely filled $d$ bands cause an attractive molecule-surface interaction at close distances and therefore no picture invocing a particular role of the $d$ holes applies to the $\mathrm{H}_{2}$-metal systems.

In summary, we have presented the first density functional theory calculation of dissociative adsorption of $\mathrm{H}_{2}$ on a metal alloy $\mathrm{NiAl}(110)$. The alloy is found to act chemically very locally, exhibiting largely varying barrier heights over $\mathrm{Ni}$ and $\mathrm{Al}$ sites. Both barriers being con-

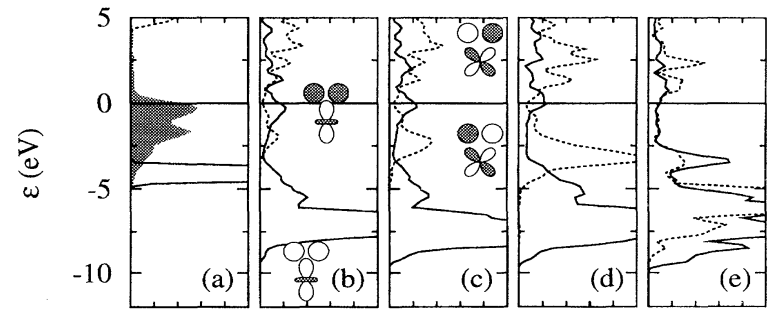

Projected DOS (arb. units)

FIG. 6. $\mathrm{H}_{2}$ dissociation over $\mathrm{Ni}(111)$ : Bonding, $n_{\sigma_{g}}(\varepsilon)$, (solid curve) and antibonding, $n_{\sigma_{u}^{*}}(\varepsilon)$, (dashed curve) projected DOS. The chosen $(b, Z)$ coordinates are (a) $(0.8,6 \AA)$, (b) $(0.8,2.0 \AA)$, (c) $(0.9,1.75 \AA),(\mathrm{d})(1.2,1.5 \AA)$, and (e) $(2.5$, $0.9 \AA)$. The position of the $d$ bands at the clean surface is indicated by the shaded area in (a).

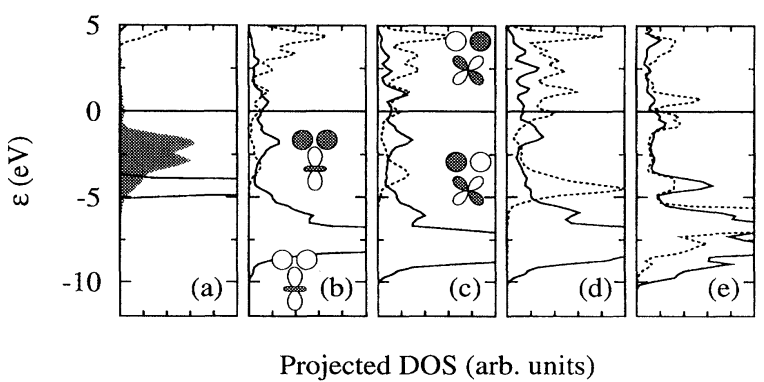

FIG. 7. Same as Fig. 6 but for $\mathrm{H}_{2}$ dissociation over $\mathrm{Cu}(111)$.

siderable. The barrier difference is explained in terms of the covalent coupling between both the bonding and the antibonding molecular orbital and the Ni $3 d$ states. This chemical picture also expounds the difference in $\mathrm{H}_{2}$ dissociation barriers observed on pure $\mathrm{Ni}$ and $\mathrm{Cu}$ surfaces.

We thank M.H. Cohen, K.W. Jacobsen, and J.K. Nørskov for fruitful discussions. B.H. gratefully acknowledges support from the Alexander von Humboldt Foundation and the Danish Research Councils. CAMP is sponsored by The Danish National Research Foundation.

[1] R. Hoffmann, Rev. Mod. Phys. 60, 601 (1988).

[2] J. K. Nørskov, Rep. Prog. Phys. 53, 1253 (1990).

[3] G. A. Somorjai, Introduction to Surface Chemistry and Catalysis (John Wiley \& Sons, New York, 1994).

[4] S. C. Lui et al., Phys. Rev. B 42, 1582 (1990).

[5] B. I. Lundqvist, O. Gunnarsson, H. Hjelmberg, and J. K. Nørskov, Surf. Sci. 89, 196 (1979).

[6] J. K. Nørskov, J. Chem. Phys. 90, 7461 (1989).

[7] J. Harris and S. Andersson, Phys. Rev. Lett. 55, 1583 (1985).

[8] J.P. Perdew et al., Phys. Rev. B 46, 6671 (1992).

[9] B. Hammer, K.W. Jacobsen, and J. K. Nørskov, Phys. Rev. Lett. 70, 3971 (1993).

[10] D. M. Ceperley and B. J. Alder, Phys. Rev. Lett. 45, 566 (1980); J. P. Perdew and A. Zunger, Phys. Rev. B 23, 5048 (1981).

[11] B. Hammer, M. Scheffler, K.W. Jacobsen, and J. K. Nørskov, Phys. Rev. Lett. 73, 1400 (1994).

[12] A. Gross, B. Hammer, M. Scheffler, and W. Brenig, Phys. Rev. Lett. 73, 3121 (1994).

[13] M. H. Kang and E. J. Meele, Phys. Rev. B 36, 7371 (1987).

[14] A. Hanbicki, A. P. Baddorf, E. W. Plummer, B. Hammer, and M. Scheffler, Surf. Sci. (to be published).

[15] P. Kratzer, B. Hammer, and J. K. Nørskov, Chem. Phys. Lett. 229, 645 (1994).

[16] E. Pehlke and M. Scheffler, Phys. Rev. Lett. 74, 952 (1995).

[17] H. J. Robota et al., Surf. Sci. 155, 101 (1985).

[18] All atomic wave functions are truncated at 2 bohrs in order to lower their overlap.

[19] D. M. Newns, Phys. Rev. 178, 1123 (1969).J. K. Nørskov, A. Houmøller, P. Johansson, and B. I. Lundqvist, Phys. Rev. Lett. 46, 257 (1981).

[20] J. K. Nørskov, A. Houmøller, P. Johansson, and B. I. Lundqvist, Phys. Rev. Lett. 46, 257 (1981). 


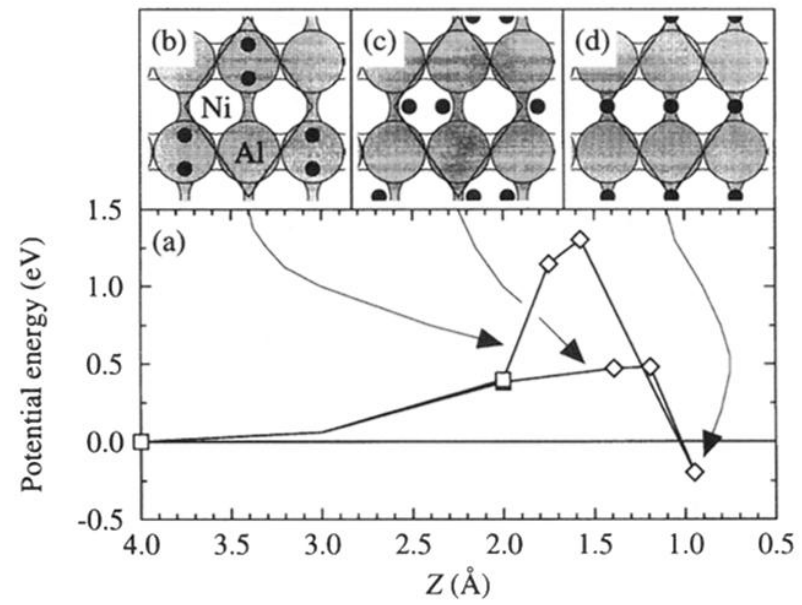

FIG. 1. (a) The potential energy of $\mathrm{H}_{2}$ dissociating over $\mathrm{NiAl}(110)$ versus the height $Z$ of the $\mathrm{H}_{2}$ center of mass from the plane of the unrelaxed surface atomic position. The upper (lower) curve is a guide to the eye connecting points for $\mathrm{H}_{2}$ over the $\mathrm{Al}(\mathrm{Ni})$ site. The geometries have been optimized with respect to the $\mathrm{H}-\mathrm{H}$ separation $b$ at fixed $Z(\square)$ or with respect to $Z$ at fixed $b(\diamond)$. (b) $\mathrm{H}_{2}$ (• pairs) over the $\mathrm{Al}$ sites (shaded circles) of the $\mathrm{NiAl}(110)$. The $c(2 \times 2)$ unit cell is indicated. (c) $\mathrm{H}_{2}$ over the $\mathrm{Ni}$ sites (open circles). (d) $(1 \times 1)$ $\mathrm{H} / \mathrm{NiAl}(110)$. 